Franziska Espinoza \& Sabine Mommartz

\title{
Sehnsucht Agilität
}

\section{Journal 맘. Psychologie} 29. Jahrgang 2021 - Hett 1

Aglile Organisationen Versuch einer kritischen Bestandsautnahm $-1$

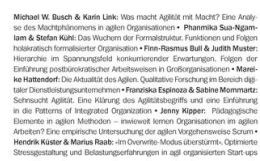

Journal für Psychologie

29. Jahrgang, Nr. 1, 2021, Seite 120-146

Psychosozial-Verlag

DOI: 10.30820/0942-2285-2021-1-120 


\section{Impressum}

Journal für Psychologie

Theorie - Forschung - Praxis

Zeitschrift der Neuen Gesellschaft

für Psychologie (NGfP)

www.journal-fuer-psychologie.de

ISSN (Online-Ausgabe): 2198-6959

ISSN (Print-Ausgabe): 0942-2285

29. Jahrgang, 2021, Heft 1

Herausgegeben von Andrea Birbaumer und Ralph Sichler

https://doi.org/10.30820/0942-2285-2021-1

ISBN der Print-Ausgabe: 978-3-8379-8354-8

ViSdP

Die HerausgeberInnen; bei namentlich

gekennzeichneten Beiträgen die AutorInnen.

Namentlich gekennzeichnete Beiträge stellen nicht in jedem Fall eine Meinungsäußerung der

HerausgeberInnen, der Redaktion oder des Verlages dar.

\section{Herausgebende}

Mag. Andrea Birbaumer, Wien · Dr. Martin Dege, Berlin · Dr. Peter Mattes, Berlin/Wien · Prof. Dr.

Günter Mey, Magdeburg-Stendal/Berlin · Dr. Aglaja

Przyborski, Wien • Paul Sebastian Ruppel, Bochum .

Univ.-Doz. Dr. Ralph Sichler, Wiener Neustadt · Dr.

Anna Sieben, Bochum/Jülich · Prof. Dr. Thomas

Slunecko, Wien

\section{Wissenschaftlicher Beirat}

Prof. Dr. Molly Andrews · Prof. Dr. Thea Bauriedl . Prof. Dr. Jarg Bergold · Prof. Dr. Klaus-Jürgen Bruder • Prof.

Dr. Stefan Busse · Prof. Dr. Tanja Eiselen • Prof. Dr. Jörg Frommer · Prof. Dr. Heiner Keupp · Prof. Dr. Carlos Kölbl · Prof. Dr. Helmut E. Lück · PD Dr. Günter Rexilius · Prof. Dr. Dr. h.c. Wolff-Michael Roth • Prof. Dr. Christina Schachtner - Prof. Dr. Rudolf Schmitt . Prof. Dr. Ernst Schraube • Prof. Dr. Margrit Schreier . Prof. Dr. Hans-Jürgen Seel · Dr. Michael Sonntag . Prof. Dr. Hank Stam · Dr. Irene Strasser, Klagenfurt · Prof. Dr. Dr. Wolfgang Tress • Prof. Dr. Jaan Valsiner - Dr. Barbara Zielke · Prof. Dr. Dr. Günter Zurhorst

\section{Erscheinen}

Halbjährlich als Organ der Neuen Gesellschaft für Psychologie (NGfP) als Open-Access-Publikation und parallel als Print-Ausgabe.

\author{
Verlag \\ Psychosozial-Verlag \\ Walltorstraße 10 \\ D-35390 Gießen \\ info@psychosozial-verlag.de \\ www.psychosozial-verlag.de
}

\section{Abonnentenbetreuung}

aboservice@psychosozial-verlag.de

\section{Bezug}

Jahresabonnement 49,90€ (zzgl. Versand)

Einzelheft 29,90€ (zzgl. Versand)

Studierende erhalten gegen Nachweis 25\% Rabatt, Mitglieder der NGfP erhalten 30\% Rabatt auf den Preis des Jahresabonnements.

Das Abonnement verlängert sich um jeweils ein Jahr, sofern nicht eine Abbestellung bis acht Wochen vor Beendigung des Bezugszeitraums erfolgt.

\section{Anzeigen}

Anfragen richten Sie bitte an den Verlag:

anzeigen@psychosozial-verlag.de

DieZeitschrift Journal für Psychologie wird regelmäßig in der Publikationsdatenbank PSYNDEX des Leibniz-Institut für Psychologie/Leibniz Institute for Psychology (ZPID) erfasst.

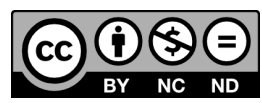

Die Beiträge dieser Zeitschrift sind unter der Attribution-NonCommercial-NoDerivatives 4.0 International Lizenz (CC BY-NC-ND 4.0) lizensiert. Diese Lizenz erlaubt die private Nutzung und unveränderte Weitergabe, verbietet jedoch die Bearbeitung und kommerzielle Nutzung. Weitere Informationen finden Sie unter: creativecommons.org/ licenses/by-nc-nd/4.0/ 


\section{Sehnsucht Agilität}

\section{Eine Klärung des Agilitätsbegriffs und eine Einführung in die Patterns of Integrated Organization}

\section{Franziska Espinoza \& Sabine Mommartz}

Journal für Psychologie, 29(1), 120-146

https://doi.org/10.30820/0942-2285-2021-1-120

CC BY-NC-ND 4.0

www.journal-fuer-psychologie.de

\section{Zusammenfassung}

Unternehmen sehen sich mit erheblichem Veränderungsdruck und sozial komplexen Problemstellungen konfrontiert, für die Agilität als Lösung gehandelt wird. Damit verbinden sie die Hoffnung, einen wenig präzise definierten Wandel zu schaffen. Im aktuellen Agilitätsdiskurs zeichnet sich vorläufig weder im Arbeitsalltag noch in der Forschung ein Konsens über den Begriff ab. Dieser bietet eine Projektionsfläche, auf der unterschiedliche Weltsichten mit großer Wucht und wenig Reflexion aufeinanderprallen. Nach einer Analyse des Begriffs aus einer kognitiv-linguistischen Perspektive und der Untersuchung der Kernversprechen, die sich seit dem Beginn des Agilitätsdiskurses im ausgehenden 20. Jahrhundert mit dem Begriff verbinden, wird ein strukturierter Reflexionsprozess, die Patterns of Integrated Organization, vorgestellt. Diese dienen zur Klärung der auf den Begriff projizierten Sehnsucht und damit als Entscheidungshilfe für eine Organisationsgestaltung im Einklang mit bewusst verhandelten Standpunkten.

Schlüsselwörter: Agilität, Organisationsentwicklung, Pattern Language, Selbstorganisation

Summary

The Promise of Agility

A Clarification of the Term Agility and an Introduction to Patterns of Integrated Organization

Companies are confronted with considerable pressure for change and socially complex problems, for both of which agility seems to promise remedy. By introducing agile structures, companies hope to bring about some change that is not defined with much precision. In the current agility discourse, there is no consensus about the term neither in everyday work nor in research. It offers a projection surface, where different worldviews collide with great force and little reflection. After a cognitive-linguistic analysis of the term in a historical context, 
a structured reflection process in the form of Patterns of Integrated Organization is presented, which serves to clarify the longing projected on the term and thus provides a decisionmaking tool for an organizational design in accordance with consciously negotiated points of view.

Keywords: Agility, organizational development, Pattern Language, self-organization

\section{Einleitung: Das Phänomen Agilität}

Das Phänomen Agilität ist vielschichtig, der Begriff schillernd, das Thema heiß. Agile Ansätze sind »in der Praxis weitreichend angekommen « (Komus 2020). Es gibt kaum noch Unternehmen, die nicht bereits Erfahrungen mit agilen Arbeitsmethoden besitzen (14th Annual State of Agile Report 2020) und der Entwicklung einer agilen Organisation hohe Priorität einräumen (Denning 2019). Gleichzeitig schätzen sich nur wenige Firmen als »sehr agil « ein (ebd.). In diesem Raum zwischen Anspruch und Wirklichkeit ist eine oft diskutierte Frage, inwiefern und in welcher Form Agilität in der Praxis tatsächlich gelebt wird (vgl. z. B. Maurer und Treutner 2020). In einem unternehmerischen Umfeld, in dem sowohl der Druck als auch der Anspruch »agil « zu sein so allgegenwärtig sind, wird $\gg$ oft mit dem Begriff um sich geworfen, ohne dass man sich über dessen Bedeutung verständigt « (Denning 2019). Es gibt auch zunehmend Stimmen, die von »fake agile « (ebd.) oder »faux-agile «, » agile that's just the name, but none of the practices and values in place « (Fowler 2018) sprechen.

Um »echte « von »falscher « Agilität zu unterscheiden oder eine Aussage zu treffen, wie »agil « Unternehmen vergleichsweise sind, braucht es - im besten Fall - eine konsensfähige Auslegung des Begriffs der Agilität, oder zumindest eine Klärung des eigenen Verständnisses. Ein Konsens über den Agilitätsbegriff zeichnet sich jedoch vorläufig weder in der Forschungsliteratur noch im Praxisalltag ab. Bei der Sichtung und dem Vergleich agiler Frameworks findet sich keine allgemein akzeptierte Definition (z. B. Sherehiy et al. 2007), und die verwendeten Agilitätskonzepte sind vieldeutig (z. B. Wendler 2013). Im Begriff fließen unterschiedlichste Organisationstheorien und Theorieströmungen zusammen (Förster und Wendler 2012; Brückner und Ameln 2016). »Agilität assimiliert sowohl eine Vielzahl bestehender älterer Konzepte als auch eine Anzahl passender neuer Konzepte, die erst entwickelt wurden. Diese Anpassungsfähigkeit erklärt möglicherweise die Schwierigkeit der Wissenschaftler, sich auf eine gemeinsame Agilitätsdefinition zu einigen « (Förster und Wendler 2012). Wenn man Führungskräfte befragt, die ihre Unternehmen agil umgestalten, betonen diese, dass große »Deutungsunklarheit und semantische Überstrapazierung « herrsche (Majkovic et al. 2019). Dennoch versprechen sich die meisten Unternehmen von einer 
Agilisierung die Lösung für die unterschiedlichen wirtschaftlich und sozial komplexen Herausforderungen, mit denen sie konfrontiert sind. Der vielfach erwartete Rückgang des Agilitätsbooms in der Unternehmenspraxis und damit auch in der Organisationsberatung (z. B. Kühl 2017) zeichnet sich bisher nicht ab.

Welche Kernversprechen soll das Konzept Agilität also einlösen? Welche Erwartungen und Hoffnungen sind mit dem Begriff verbunden? Eine Klärung dieser Frage ist im Kontext von organisationalen Transformationsvorhaben von hoher Praxisrelevanz. Bei der Einführung agiler Ansätze ist für Führungskräfte und Mitarbeitende genauso wie für Organisationsberater_innen wichtig, nicht nur agile Konzepte und Verfahrensweisen zu kennen, sondern die eigenen mit dem Agilitätsbegriff verbundenen Hoffnungen zu reflektieren und sich selbst im Hinblick auf die aktuellen Agilitätsdiskussionen zu verorten.

Die vorliegende Arbeit möchte zu einer fundierten und strukturierten Reflexion anregen. Sie untersucht, wie es dem Agilitätsbegriff wie kaum einem anderen Begriff der Organisationsentwicklung gelingt, die kollektive Aufmerksamkeit (Brückner und Ameln 2016) zu bündeln (Kap. 2), und geht der Frage nach, welche wichtigen Kernversprechen seit Beginn des Agilitätsdiskurses in den 1990er Jahren bis heute mit diesem Begriff verbunden werden (Kap. 3). Eine Klärung des Agilitätsbegriffs selbst und ein bewusstes Reflektieren der auf den Begriff projizierten Erwartungen sollten bei einer Einführung oder Weiterentwicklung agiler Arbeitsformen - so postuliert der Beitrag neben der inhaltlichen Auseinandersetzung mit den Agilitätskonzepten und deren Herausforderungen (u. a. Porschen-Hueck et al. 2020) unbedingt berücksichtigt werden. Als strukturierte Reflexionshilfe wird dazu mit den Patterns of Integrated Organization eine Entwicklungslandkarte vorgestellt, die Unternehmen als Orientierung auf dem Weg zu einer agilen oder vielleicht auf andere Weise passenden, authentischen Organisation dienen kann (Kap. 4).

\section{Der Agilitätsbegriff: »Metaphors we argue by« oder wieso wir alle agil sein wollen}

Eine » zentrale Leistung [des Agilitätskonzeptes] für die Reflexion und Bearbeitung der aktuellen Problemlagen [ist es], die Aufmerksamkeit auf die sehr aktuelle Frage der Reagibilität von Organisationen zu lenken und dabei einige spannende Diskussionsstränge zu bündeln « (Brückner und Ameln 2016). Diese Bündelung der Aufmerksamkeit ist nicht nur dem Agilitätskonzept, sondern auch dem Agilitätsbegriff selbst und dem Metaphernfeld, in dem er eingebunden ist, geschuldet.

Das Wort » agil « ist ein in unserem Kulturkreis positiv konnotiertes Adjektiv, wenn es nicht gerade seines inflationären Gebrauchs wegen zu einem Buzzword oder Unwort 
(Truscheit 2018) wird. Es ist kollektiv erstrebenswert, bis ins hohe Alter agil zu sein, und nur ungern lässt man sich sagen, das eigene Denken und Handeln sei nicht agil. Adjektive wie »lean «, » selbstorganisiert « oder » holokratisch « sind nicht auf die gleiche Weise Teil unseres persönlichen Selbstverständnisses.

Wie viele andere Lehnwörter aus dem Lateinischen geht » agil « auf das Verb » agere «, » in Bewegung setzen, treiben «, zurück. ${ }^{1}$ Wörter wie » agieren «, » reagieren «, »Aktion «, »Interaktion «, »Akteur «, »Agenda « oder »aktiv « gehören zu jenen Wörtern, deren Übertragung von einer konkreten zu einer abstrakteren Bedeutung schon lange vor der Entlehnung ins Deutsche stattgefunden hat (Deutscher 2011, 146). Der Erfahrungsbereich der Bewegung und der räumlichen Veränderung - in Bewegung setzen - wurde auf den abstrakteren Bereich - agieren, handeln, verhandeln - angewandt, um diesen kommunizierbar zu machen.

Die kognitive Metapherntheorie von Lakoff und Johnson (1980), die seit ihrer Entstehung von anderen Forschern_innen in verschiedenen Disziplinen, unter anderem auch in der Organisationspsychologie (Morgan 1997) und von den Autoren selbst (Lakoff und Johnson 1999 und 2003) präzisiert und weiterentwickelt wurde (vgl. Schmitt 2017), geht von der zentralen Annahme aus, dass wir sinnlich erfahrbare Herkunftsbereiche, wie zum Beispiel die Geschwindigkeit einer Bewegung, nutzen, um abstrakte Zielbereiche, wie zum Beispiel das Konzept der $\gg$ Intelligenz «, zu strukturieren. Eine solche Übertragung hat auch beim Adjektiv »agilis « (beweglich) stattgefunden, das über das französische »agile« erst im 17. Jahrhundert ins Deutsche und Englische ${ }^{2}$ Eingang gefunden hat. Die Fähigkeit, sich flink und rasch zu bewegen, wird auf das Denken übertragen. Hat jemand einen » agile mind «, bedeutet das, intelligent zu sein, » to think quickly, solve problems and have new ideas ${ }^{3}$.

Dass es sich hier überhaupt um eine Bedeutungsübertragung bzw. eine Metaphernbildung handelt, ist uns oft nicht ersichtlich. Die Metaphern, in denen wir sprechen und denken, sind uns meist so vertraut, dass wir sie kaum als solche wahrnehmen. Metaphern drücken eine allgemein akzeptierte, zur Konvention geronnene Weltsicht aus: »The > fixed truths $<$ of our culture are nothing but metaphorical understandings that have become conventionalized to the point where their metaphoricity is forgotten « (Johnson 1981, zit. nach Schieder 2006).

Wenn wir sagen »die Aktien steigen « (Moser 2000), »die Zeit fliegt « oder » ein Unternehmen löst sich vom Feld « und »punktet « (Bröckling 2014), sprechen wir in Metaphern. Diese Aussagen sind Ausdruck unseres Denkens. Wir begreifen »oben als mehr «, $\gg$ Zeit als bewegliches Objekt «, und $\gg$ das wirtschaftliche Leben als sportlichen Wettkampf « (u. a. Lutter 2016; Bröckling 2014). Im letztgenannten Beispiel dient das Prinzip der »Konkurrenz als übergreifendes Realitätsschema, um die Wirklichkeit zu begreifen, sich an ihr zu orientieren und das eigene Handeln auszurichten « (Bröckling 2014). 
Metaphern und Metaphernfelder sind nicht beliebig, sondern kulturell bedingt. Metaphern sind ein »Umschlagplatz sozialer Tradition « (Pielenz 1993, 108). Sie erheben Anspruch auf Objektivität, legitimieren und sanktionieren. Es ist theoretisch möglich, sich das Marktgeschehen nicht in kriegerischen Termini oder in Bildern des Leistungssportes vorzustellen. Man könnte einen anderen Aspekt des Sportes hervorheben, wie die »Lust an körperlicher Bewegung « oder »das zweckfreie Spiel « (Bröckling 2014), oder ein anderes Metaphernfeld nutzen und sich das wirtschaftliche Leben als Tanzform vorstellen. Doch wird man sich damit wohl kaum anderen mitteilen und verständlich machen können (vgl. Lakoff und Johnson 1980). Mit ihren »beiden kognitiven Mechanismen > highlighting und hiding « (Noack Napoles 2020) heben Metaphern bestimmte Aspekte eines Konzeptes heraus und verdeutlichen diese, während sie andere vernachlässigen oder sogar deren Wahrnehmung verhindern.

Die Kernversprechen, die wir im Folgenden untersuchen, sind entscheidend durch die Metaphorik des Agilitätsbegriffs und die dazugehörenden Metaphernfeldern geprägt.

\section{Projektionsfläche Agilität: Die Kernversprechen}

Seit dem Beginn des Agilitätsdiskurses in den 1990er Jahren des letzten Jahrtausends haben drei Publikationen mit ihren jeweiligen Agilitätsversprechen größte öffentliche Aufmerksamkeit erhalten. 1991 erfolgte die Veröffentlichung des »21ST Century Manufacturing Enterprise Strategy Reports « (Nagel 1991) des Iacocca Institutes der Lehigh University, der im Folgenden Lehigh-Report genannt wird (Kap. 3.1). Der Bericht postulierte eine agile Wende mit einer Argumentationslogik, die bis heute Verwendung findet. Im Jahr 2001 wurde das »Agile Manifest « (Beck et al. 2001) veröffentlicht, welches oft als der Ursprung der agilen Bewegung betrachtet wird (Kap. 3.2). 2014 erschien das Buch Reinventing Organizations (Laloux 2014), welches mit seinen Recherchen zu einem organisationalen Paradigmenwechsel dem Agilitätsdiskurs eine zusätzliche Dimension hinzufügte (Kap. 3.3). Auch wenn der Agilitätsbegriff bei Laloux nicht vorkommt, wurde der Autor dennoch als »Vordenker der agilen Organisation « und »Vertreter des Agilitätskonzeptes « rezipiert (z. B. Brückner und Ameln 2016).

\subsection{Das erste Agilitätsversprechen (1991): Agilität als Waffe im Wettbewerb}

Den traditionellen amerikanischen Unternehmen fehlte es an der Fähigkeit, sich rasch genug an veränderte Marktbedingungen anzupassen, so lautete die selbstkritische Analyse von Industrievertretern gegen Ende des letzten Jahrtausends: »Agility was born 
1991 when an industry group observed that the increasing rate of change in the business environment was outpacing the adaptability of traditional manufacturing organizations « (Dove 1994).

Die amerikanische Fertigungsindustrie mit ihren Produktionsmethoden und der wissenschaftlichen Betriebsführung, das ursprünglich von Taylor entworfene Konzept minutiöser Prozesssteuerung, schien an ihre Grenzen zu stoßen. Das amerikanische Verteidigungsministerium zeigte sich besorgt. Erst kurze Zeit zuvor hatten sich die USA und die UdSSR auf das Ende des atomaren Wettrüstens und des Kalten Krieges geeinigt. Viele amerikanische Firmen kämpften mit finanziellen Schwierigkeiten, während die EU nach dem Fall der Berliner Mauer kräftig in ihre Industrie investierte (Nagel 1991; De Meyer und Ferdows 1991). Die japanischen Autobauer schockierten mit ihrer $»$ Lean Production «, einem standardisierten, weniger hierarchisch funktionierenden Produktionssystem. Überhaupt stellten die prozessualen und technischen Innovationen Japans für die Industrie der westlichen Welt einen »kulturellen Schock « dar. Das European Management Journal argumentierte, dass die Arbeitsweise der japanischen High-Performance-Teams aus kulturellen Gründen schwierig nachzuahmen sein werde (Karlsson 1989). Besonders hohe Beachtung hatte in diesem Zusammenhang 1986 ein Artikel der japanischen Wissenschaftler Takeuchi und Nonaka in der Harvard Business Review gefunden, der als Antwort auf $\gg$ today's fast-paced, fiercely competitive world of commercial new product development « (Takeuchi und Nonaka 1986) einen innovativen, holistischen Ansatz in der Produktentwicklung von Firmen wie Honda, Kodak und Fuji-Xerox beschrieb, mit dem der auch in Japan vorherrschende Taylorismus überwunden und Geschwindigkeit und Flexibilität gewonnen werden konnte.

Das US-Verteidigungsministerium gab deshalb zur Wahrung der gemeinsamen Interessen der Industrie und der Regierung eine Studie in Auftrag. Unter der Führung des Iacocca Institutes der Lehigh University wurden mit einer Vielzahl von Unternehmens- und Regierungsvertretern einen ganzen Sommer lang Workshops durchgeführt, um einen nationalen Konsens zur Lage der Nation zu finden und Zukunftsszenarien zu formulieren. Das Ergebnis wurde im Lehigh-Report veröffentlicht. Damit sei, so reflektierte ein Mitverfasser, das Konzept des agilen Industriebetriebes geboren, mit »agility « as »the single most important characteristic an enterprise will have when entering the $21^{\text {st }}$ century « (Dove 1992). Die amerikanischen Unternehmen sollten, so die dringliche Botschaft des Berichts, mit einer agilen Wende ihre Wettbewerbsfähigkeit und damit Amerikas wirtschaftliche und politische Hegemonie zurückerobern. Ohne eine komplette Umgestaltung von »Technologien, Management-Strukturen und sozialen Werten « (Nagel 1991) sei das Aufrechterhalten des amerikanischen Lebensstandards nicht mehr möglich. Mit der Vision der agilen Unternehmung wollte man eine »mächtige Waffe im Wettbewerb« (ebd.; Hervorh. d. A.) schaffen. Das war das Kernversprechen. 
Im Bericht wurde die Dringlichkeit, mit der dieses Kernversprechen durch die amerikanischen Firmen eingelöst werden sollte, und der Geltungsanspruch der postulierten Umsetzungsagenda mit der Verwendung von Metaphern und wirkungsvollen Argumentationsmustern auch sprachlich legitimiert. Einem Mantra gleich wiederholten die Verfasser des Lehigh-Reports, dass sie sich »in an age of continuous and unrelentig change « (Dove 1994) befänden. Der Wandel ist eine unaufhörliche und unabwendbare Bewegung. Dies ist eine kollektive Weltsicht, die uns so vertraut ist, dass wir nicht über deren Wahrheitsgehalt nachdenken müssen. »Zeit ist ein unaufhaltsames bewegliches Objekt « und so ist auch »Veränderung ein unaufhaltsames bewegliches Objekt «. Es ist eine schlüssige Argumentation, dass ein hereinbrechender Wandel, den man als unaufhaltsam erfährt, ein Aufruf zu Bewegung und zur Anpassung der eigenen Beweglichkeit und Veränderungsgeschwindigkeit darstellt: $\gg$ In an age of continuous and unrelentig change, rapid response must be a core competency « (Dove 1994).

Die Situationsanalyse, die Dove in seiner Aussage entwirft, entspricht einer kollektiven Betrachtungsweise, mit Ausnahme vielleicht des Attributes » unerbittlich «, welches in diesem Zusammenhang auf die Ernsthaftigkeit der Lage hinweist und ihr den nötigen Spin gibt: Man hat es hier mit einem unpersönlichen Gegner zu tun, der kein Erbarmen zeigt. Die Erfahrung des Wandels als unaufhaltsame, oft unberechenbare Bewegung verdichtet sich im Zeitalter der Moderne zur beschleunigten Bewegung in der Zeit. Die technologische Beschleunigung, die Beschleunigung des sozialen Wandels und die Beschleunigung des Lebenstempos sind allgegenwärtig, einem » unerbittlichen « Feind gleich, der einem Ruhe und Gelassenheit nimmt. Sie gehört zum kollektiven Lebensgefühl der postmodernen, gehetzten Menschen (vgl. Rosa 2013, 16-17). Die geteilte Erfahrung der Beschleunigung ist für sich genommen noch keine handlungswirksame Argumentation. Doch in der Zeit des ausgehenden Kalten Krieges und des Wettrüstens ist dieses Lebensgefühl eng verknüpft mit der Vorstellung des » wirtschaftlichen Lebens als Krieg « (Lutter 2016). Diese Erfahrung kombiniert aus Beschleunigung, Wettrüsten und Wettbewerb ist ein mächtiges Argumentationsmuster, das bis heute seine Wirkung nicht verloren hat. >Nicht-Agilität< ist undenkbar, denn die Parole lautet: »Stillstand ist Tod « (Schmitz-Ohrndorf 2016), »Wer überleben will, sollte auf Veränderung setzen « (Hamacher 2018), »Wer agil ist, überlebt! « (Newton 2017).

\subsection{Das zweite Agilitätsversprechen (2001): Agilität »based on people and collaboration«}

Die Softwareentwickler, die in jenen amerikanischen Unternehmen arbeiteten, die sich nach der Veröffentlichung des Lehigh-Berichts einem neu gegründeten Agilen Forum angeschlossen hatten, waren mit dem Begriff und der Argumentation der Dringlichkeit 
einer agilen Wende wohl vertraut (Beedle 2017). Der 1986 in der Harvard Business Review erschienene Artikel »The New New Product Development Game« (Takeuchi und Nonaka 1986) löste bei den Ingenieuren nicht Besorgnis und Wettbewerbsstress, sondern große Begeisterung aus.

Der Inhalt des Artikels, in dem anhand einer Metapher aus dem Sport ein Systemwechsel in der Produktionsentwicklung beschrieben wurde, inspirierte Jeff Sutherland direkt zur Modellierung von Scrum (Sutherland 2011), dem heute am häufigsten genutzten agilen Ansatz (Komus 2020; 14th Annual Agile Report 2020). Man sollte sich die Zusammenarbeit nicht mehr wie einen sequenziellen Staffellauf von Einzelkämpfern vorstellen, sondern wie Rugby. Die Spieler sollten sich gemeinsam im $\gg$ Scrum «, im »Gedränge «, um einen Ball scharen, das heißt um einen Auftrag herum organisieren $\gg$ motivated by transcendent purpose, and engaged in cross learning « (Sutherland 2011) und autonom und in kurzen Iterationszyklen hochwertige Software entwickeln. Für die Softwareingenieure mit ihren Erfahrungen in großen, nach rigiden Planungsmethoden geführten und oft scheiternden Projekten war dies ein lang ersehnter Systemwechsel. Pioniere unter ihnen hatten begonnen, mit leichtgewichtigen Methoden, eXtreme Programming, Softwareentwicklungspatterns (siehe Kap. 4) und selbstbestimmteren Zusammenarbeitsformen zu experimentieren. Als einige von ihnen beschlossen, sich mit ihren Kollegen über ihre Pionierarbeit auszutauschen, lag auch ein Hauch Revolution in der Luft (Beedle 2017).

Die Konferenz fand mit 17 Ingenieuren im Februar 2001 in Snowbird in Utah statt. So kontrovers die Softwareentwickler ihre unterschiedlichen neuen Zusammenarbeitsformen, Projektmanagementmethoden und Programmiertechniken diskutierten, so einfach konnten sie sich in einem gemeinsamen Prozess auf vier grundlegende Haltungen einigen, die sie als Manifest veröffentlichten. Sie wollten damit bessere Wege erschließen, Software zu entwickeln. Individuen und Interaktion sollten vor Prozessen, Zusammenarbeit mit Kunden vor Vertragsverhandlungen, funktionierende Software vor umfassender Dokumentation und Reagieren auf Veränderungen vor dem Befolgen eines Planes stehen (Beck et al. 2001). Damit versprachen sie sich im Kern eine Zusammenarbeit, die stärker auf Vertrauen, unbürokratischen Organisationen und menschenund kommunikationsorientierten Regeln (Cockburn 2002) beruhte. Das Manifest » [is] promoting organizational models based on people, collaboration, and building the types of organizational communities in which we would want to work « (Highsmith 2001; Hervorh. d. A.). Mit einer Prise Humor sagten sie, ihr agiler Ansatz bestehe darin, »in Frieden und Harmonie zusammenzukommen, jede Woche Software zu liefern und dabei immer eine Retrospektive zu machen, um zu sehen, wie man sich verbessern könne « (Fowler 2018). Der Begriff » agil « als Oberbegriff des Manifests fand sich eher zufällig. So berichtet Mike Beedle (Beedle 2017), der damals bei IBM arbeitete und mit der Arbeit des Agilen Forums und dem Buch der Verfasser des Lehigh Berichts Agi- 
le Competitors and Virtual Organizations (Goldmann 1994) vertraut war, er habe den Vorschlag eingebracht, das Wort » agil « zu verwenden. Nachdem sie » adaptive «, »essential «, »lean « und »lightweight « verworfen hatten, unter anderem weil niemand ein »Leichtgewicht « (Beedle 2017) sein wollte, entschieden sie sich rasch für » agile «. Beedle (2017) erinnert sich, dass »Martin Fowler said: > well Mike, the only problem when we use »agile«, we are going to be successful. And everybody laughed and we went with $\gg$ agil $\ll .<\ll$

Die Veröffentlichung des Manifests löste eine große Resonanzwelle aus. Die Bekanntmachung traf auf eine kollektive Sehnsucht, »an untapped undercurrent of desire (Marick 2011). Viele Menschen in ähnlichen Arbeitsverhältnissen mit den gleichen Emanzipationsbestrebungen teilten die Gefühlslage der Softwareingenieure. Mit der Wahl des Begriffs »Agilität«, zehn Jahre nach der Veröffentlichung des Lehigh-Berichts, verhalfen sie ihren »leichtgewichtigen « Projektmanagementmethoden zum Durchbruch, auch dort, wo diese zuvor von Führungskräften als zu »extrem « abgewehrt worden waren (vgl. Grenning 2017). Ihr Agilitätsbegriff war mit dem Kernversprechen der vertrauensvollen Zusammenarbeit in einer Gemeinschaft von Gleichgesinnten verbunden. Das vorangegangene und das neue Kernversprechen gehörten einer Ideenordnung an, die unterschiedlicher nicht sein konnte - dies war ja gerade das Revolutionäre und Attraktive an den Prinzipien des Manifests.

Das Prinzip der Orientierung am Kunden erwies sich als ein Prinzip der radikalen Orientierung am Auftrag (Baecker 2017), das den Softwareingenieuren zu einer Emanzipation von einem rigiden Führungssystem verhalf. Die Einführung einer agilen Produktentwicklung führte in Ansätzen dazu, dass sich das Management mit der $\gg$ Abschaffung der klassischen formalen Hierarchie der Rangordnung auch selbst abschafft $[\mathrm{e}]$, beziehungsweise, vorsichtiger formuliert, sich reformatiert $[\mathrm{e}] \ll$ (Baecker 2017). Gleichzeitig aber, und dies war der eigentlich tragische Umstand, bedienten die Ingenieure mit ihrer Begriffswahl das etablierte Management und dessen ein Jahrzehnt zuvor verkündete agile Wettbewerbsstrategie. Auch wenn die Namensgebung scheinbar beiläufig geschah, mussten die Ingenieure - so lässt ihr Lachen vermuten - doch kollektiv gewusst oder zumindest geahnt haben, welche Dynamik sie damit auslösen würden (vgl. Fowler, 2006; Grenning 2017).

Der ideologische Wechsel zeigt sich auch in der Verwendung von Metaphern in den Texten der Co-Autoren des Manifests, welche die Metaphern des Wettrüstens zwischen Unternehmen zu Metaphern des Mannschaftsspiels umdeuten: »Winning in business increasingly involves winning at the software development game, winning at the game depends on understanding the game being played [...]. We have seen that software development is a group game, which is goal seeking, finite, and cooperative « (Cockburn 2002; Hervorh. d.A.). Cockburn verwendet sorgfältig die allgemein gebräuchlichen Aspekte der Sportmetaphern, »die sich auf Konstellationen des Wettkampfes bezie- 
hen « (Bröckling 2014), und verbindet diese mit solchen Bereichen, die um das Prinzip der Kollegialität kreisen. Was nach einer Erfolgsgeschichte aussah und immer noch aussieht - die Befreiung aus den Fesseln des bürokratischen, rigiden Managementsystems und die Einführung von Prinzipien einer menschenorientierten, vertrauensvollen Gestaltung der Zusammenarbeit - erhielt viel Unterstützung. Doch die »Befreiten« wurden sogleich wieder »Sklaven ihres Erfolges «. Die neuen Zusammenarbeitsformen erwiesen sich zwar tatsächlich als produktiver, performanter und wettbewerbsfähiger. Sie waren deshalb auch agil im Sinne des ersten Kernversprechens. Doch sie wurden oft ohne die neue, intendierte Werthaltung eingeführt. »Dark Scrum « (Jeffries 2016; Fowler 2018) nannten die Manifesto-Verfasser dieses Phänomen. Wurde Scrum nicht im Sinne des zweiten Kernversprechens gelebt, schien es » nur zu oft die Leute zu unterdrücken « (Jeffries 2016), und das Wort » agil « wurde gegen sie und entgegen den Prinzipien des Manifestes genutzt (Fowler 2018).

Die Spannungen und die Konflikte, die dieses Aufeinandertreffen der beiden Kernversprechen auslöste, sind bis heute die gleichen geblieben (u. a. Porschen-Hueck et al. 2020).

\subsection{Das dritte Agilitätsversprechen (2014): Die Sehnsucht nach sinnhafter Arbeit}

13 Jahre später erschien ein Buch, welches der Agilitätsdiskussion noch einmal einen neuen Dreh geben sollte. Die ersten Anzeichen einer verborgenen Gegenströmung, die Marick (2011) bei der Veröffentlichung des »Agilen Manifests « wahrnahm, waren 2014 zu einem nicht übersehbaren Strom geworden. Das Buch Reinventing Organizations (Laloux 2014) wurde über Nacht zum Bestseller. Laloux stellt darin zwölf innovative Unternehmen vor, die unabhängig voneinander mit neuen Zusammenarbeitsformen experimentierten. Ihre Sehnsucht galt Werten wie Freiheit und Autonomie, Ganzheit und Selbstentwicklung. Bei ihrer Suche ließen sie sich genauso durch die agile Softwareentwicklung inspirieren wie durch Theorien der Selbstorganisation. Was ihrem »Purpose« diente, war ihnen willkommen. Weder Laloux noch die Unternehmen selbst bezeichneten ihre organisationalen Praktiken als agil. Doch da der Begriff unter anderen auch Diskussionsstränge um Selbstorganisation, Demokratisierung und Enthierarchisierung (Brückner und Ameln 2016) beinhaltete, wurde Laloux als »Vordenker der agilen Organisation « (ebd.) rezipiert. Die im Buch beschriebenen innovativen $\mathrm{Zu}$ sammenarbeitsformen wurden mit dem Agilitätsbegriff in Verbindung gebracht: »Teal is the New Agile « (Quartel 2019).

Einige der neuen Organisationsformen, wie Holacracy (Robertson 2015), wurden direkt mit dem Agilitätskonzept verknüpft (u.a. Sutherland 2014) und auch mit den 
Kernversprechen der Leistungssteigerung im Wettbewerb in Bezug gebracht, bei anderen, wie bei Buurtzorg, einem niederländischen ambulanten Pflegedienst, blieb diese Verbindung aus. Dies hieß keinesfalls, dass dieses Unternehmen nicht prosperierte. Buurtzorg wuchs nach seiner Gründung von vier auf 15.000 Betreuungskräfte und wurde zum größten Anbieter ambulanter Pflege in den Niederlanden. Buurtzorg senkte die Kosten des holländischen Gesundheitssystems um 40\%. Als das Pflegepersonal nicht mehr unter dem Diktat der Metapher »Zeit ist Geld « arbeitete und sich zur Betreuung der Patient_innen Zeit nehmen konnte, halbierten sich die eingesetzten Pflegestunden (Johansen und Van den Bosch 2017). Im Sinne des Kernversprechens sinnhafter Arbeit und entsprechend seiner Vision einer ganzheitlichen Pflege unterstützte Buurtzorgs Gründer Jos de Blok alle anderen Anbieter von Pflegedienstleistungen mit dem Ziel, diese genauso erfolgreich zu machen. Wettbewerb und Performancesteigerungen sind in dieser Unternehmenswirklichkeit keine zielgebenden Größen (Laloux 2014).

»Die Gründer von evolutionären Organisationen nutzen andere Metaphern für die Arbeitsumgebung, die sie gestalten möchten. Mit überraschender Häufigkeit sprechen sie über ihre Organisationen als lebende Organismen oder lebendige Systeme [...]. Diese Metapher eröffnet neue Möglichkeiten. Stellen Sie sich vor, wie sich Organisationen verändern könnten, wenn wir sie nicht länger wie seelenlose, schwerfällige Maschinen gestalten, sondern sie wie Lebewesen behandeln? « (ebd., 54)

Laloux setzt den Gebrauch von Metaphern und Metaphernfeldern nicht nur in der Analyse der Entwicklungsstufen von Organisationen ein, sondern - wie es auch die Pionierunternehmen tun, über die er schreibt - als bewusste Reflexion zukünftiger Gestaltungsmöglichkeiten.

Die drei Kernversprechen aus den Jahren 1991, 2001 und 2014 unterschieden sich deutlich in den ihnen zugrunde liegenden Vorstellungen und Überzeugungen. Solange ein Begriff jedoch lebendig ist, sind wir uns selten gewahr, dass die Unklarheiten und Deutungsprobleme, denen wir im Unternehmensalltag begegnen (Majkovic 2019), aus einem »Mangel an Kohärenz zwischen diesem Begriff und der ihn ermöglichenden früheren Ideenordnung hervorgegangen ist. Begriffe haben Erinnerungen an Ereignisse, die wir vergessen haben « (Hacking 2001, 83-84). So prallen im Agilitätsbegriff unterschiedliche Weltverständnisse aufeinander:

Ein modernes, leistungsorientiertes Paradigma - mit dem Versprechen des Wettbewerberfolges durch beschleunigtes Arbeiten - trifft sowohl auf ein postmodernes, pluralistisches Paradigma, in dem Agilsein mit den Werten der Menschenorientierung und Beziehungsgestaltung gleichgesetzt wird, als auch auf ein integral-evolutionäres Paradigma, in dem agile Organisationsformen sinn- und potenzialorientierte Arbeitswelten schaffen sollen. 
Diese gleichzeitige Projektion unterschiedlichster Weltsichten auf den Agilitätsbegriff hat nicht zu unterschätzende Auswirkungen auf den Unternehmensalltag. Das Ausmaß lässt sich an der Häufigkeit und der Verve erahnen, in der im Arbeitsalltag in Internetforen über die Auslegung des Agilitätsbegriffs diskutiert wird. Es zeigt sich auch in der Ausrichtung einer ganzen Beratungsindustrie, die » agile Mindset $[s] \ll$ vermitteln will (Volkmer 2019). Vor allem aber zeigt es sich bei der Einführung agiler Organisationsformen in der betrieblichen Praxis selbst.

\section{Eine integrale Reflexionspraxis}

Der Agilitätsbegriff wird - wie anhand der drei Kernversprechen gezeigt - zwar in unterschiedlichen Kontexten jeweils neu interpretiert, doch lässt er sich gleichzeitig kaum aus den jeweils zuvor in anderen Umgebungen entstandenen Bedeutungszuschreibungen lösen. Will man deshalb ganz auf den Begriff verzichten, wie zum Beispiel Fowler bereits fünf Jahre nach der Unterzeichnung des Manifests diskutiert (Fowler 2006), besteht die Gefahr, dass ein neuer Begriff nur zusätzliche Verwirrung stiftet (ebd.) und man sich damit nicht nur der Anziehungs- und Argumentationskraft des Begriffs entledigt, sondern auch der auf den Begriff projizierten individuellen und kollektiven Versprechen. So sind mit dem Begriff auch richtungsweisende Wünsche und Zukunftsvisionen verbunden, welche in einer Phase der Neuorientierung zentral sind. Es bedarf deshalb bei der Einführung oder Weiterentwicklung agiler Ansätze nicht einer Abkehr vom Agilitätsbegriff, sondern einer bewussten Hinwendung, um das eigene Verständnis des Begriffes und den eigenen Standpunkt in Bezug auf potenzielle Veränderungen im Unternehmen radikal zu reflektieren.

Diese Reflexion stellt den Ausgangspunkt der Entwicklung zu einem agilen Unternehmen dar. Sie darf jedoch nicht nur zu Beginn eines Transformationsvorhabens erfolgen, sondern muss ein zentrales Element der täglichen Arbeit werden. Alle Beteiligten sollten sich dabei grundsätzlich bewusst machen, dass sie selbst über ihre Sprache den Dingen ihre Bedeutungen zuschreiben, und bereit sein, diese Deutungen und die damit einhergehende Sicht der Welt für die anderen Beteiligten transparent zu machen.

Die Art und Weise, wie sich die Mitglieder einer Organisation zu arbeiten erhoffen, muss anhand des Agilitätsbegriffes, aber auch unabhängig von diesem geklärt und mit den Möglichkeiten des Unternehmens abgeglichen werden. Es ist wichtig, dass Wünsche, Hoffnungen und Befürchtungen expliziert werden. Dieses Wissen dient als »Standpunkt und Ausgangspunkt « der Veränderung. Ein solcher Ausgangspunkt lässt sich auch als eine Haltung verstehen, die noch keine Richtung einschlägt und keine Position bezieht. Es ist nur ein In-sich-Ruhen. An diesem Ort ist »Stillstand nicht 
Tod «, Wandel nicht mehr zwingend eine Bewegung in eine Richtung und kein unaufhaltsames Fortschreiten. Stillstand ist hier gleichbedeutend mit einem »Zustand des relativen Gleichgewichts « (Noack 2020). Ein Gleichgewicht mit sich und dem eigenen $\gg$ Purpose « ist ein »Mittelzustand, [...] in dem man sich mit dem, was man tut und wo man es tut, eins fühlt « und wo »das Spiel freier, die Gesundheit strahlend, das Geschlechtsleben reifer und die Arbeit sinnvoller geworden ist « (vgl. ebd.). Von hier ausgehend können sich die Beteiligten mit der Frage auseinandersetzen, wie sie ihre Organisationen grundlegend neu gestalten wollen.

Wie kann in der Praxis diese Reflexion so erfolgen, dass alle Beteiligten ihren eigenen Standpunkt und ihre Veränderungsbereitschaft klären, miteinander abgleichen und ein gemeinsames Verständnis für die Unternehmensveränderung entwickeln? Es braucht dazu einen strukturierten Prozess, welcher hilft, die eigenen und die kollektiven Annahmen, Wünsche, Ängste und Abwehren bewusst zu machen.

Im folgenden Kapitel stellen wir mit den Patterns of Integrated Organization einen solchen von uns entwickelten, strukturierten Reflexionsprozess vor, der den Beteiligten ermöglicht, den eigenen Standpunkt zu klären und in der Folge weitere Leitfragen zu explizieren, die sich bei jedem organisationalen Konflikt, bei strukturellen Veränderungen und ganz besonders bei der Einführung von selbstorganisierten Arbeitsformen stellen.

Die Patterns bzw. der von ihnen unterstützte Reflexionsprozess ist nicht als Instrument zur »Agilisierung « zu verstehen, sondern als eine Entwicklungslandkarte, die es ermöglicht, eine präzise Diagnose zu stellen und eine Klärung des gemeinsamen Standpunktes als Grundlage einer potenziellen und für das jeweilige Unternehmen passenden agilen oder authentischen Organisation zu finden. Das Ergebnis dieser Diagnose bezieht sich immer auf den Standpunkt der beteiligten Mitglieder einer Organisation und ist einzigartig und nicht übertragbar.

\subsection{Eine kurze Einführung in Patterns und Pattern Languages}

Christopher Alexander veröffentlichte 1977 das bahnbrechende Buch A Pattern Language for Building. Es enthält 253 Patterns für den Bau von Städten, Gebäuden und Konstruktionen. Die Patterns sind für Laien geschrieben, die sich mit deren Hilfe selbst Umgebungen schaffen können, in denen Menschen sich frei fühlen: »[I]n a living environment, as we define it, the people who live and work there are relaxed, know that they belong there and enjoy a kind of freedom in relation to the buildings and space around them. They feel and are $>$ at home $<$ in their world $\ll^{4}$.

Christopher Alexanders Pattern Language hatte eine elektrisierende und tiefgreifende Wirkung auf unterschiedlichste Fachgebiete und ganz besonders auf die Soft- 
ware-Engineering Community. Die Co-Autoren des »Agilen Manifests «, die schon 1987 mit dem Schreiben von Patterns begonnen hatten (Vlissides et al. 1996), waren in der Welt der Patterns zu Hause. Die Erfindung des Wikis als Pattern Language durch Ward Cunningham ist unter anderem Zeuge davon (Cunningham und Mehaffy 2013).

Ein Pattern ist » at the same time a thing, which happens in the world, and the rule which tells us how to create that thing and when we must create it. It is both a process and a thing; both a description of a thing which is alive, and a description of the process which will generate that thing (Alexander 1979, 247).

Pattern Languages sind das Resultat empirischer Beobachtungen. Sie sind in der Praxis angesiedelt. Sie sind als strukturierte Reflexionshilfe gedacht und gleichzeitig als Anregung, wie man ein spezifisches Gestaltungsproblem lösen kann. Ein Gestaltungsproblem ergibt sich immer dann, wenn in einer Situation eine Spannung zwischen gegensätzlichen Kräften besteht, die nicht ausbalanciert sind.

Eine solche Spannung, die Christopher Alexander beschreibt, besteht zum Beispiel dann, wenn es in einem Raum auf der einen Seite ein Fenster gibt und auf der gegenüberliegenden, dunkleren Seite eine Sitzgelegenheit. Die Menschen, die den Raum betreten, fühlen sich in der Regel unwohl, denn sie möchten sich zwar gerne hinsetzen und gleichzeitig möchten sie lieber nahe beim Fenster sein, um das Sonnenlicht zu genießen und hinauszuschauen. Wenn man dieses Problem bei der Raumgestaltung erkannt hat, ist es einfach zu beheben, zum Beispiel indem die Sitzgelegenheit zum Fenster verschoben wird. Dies schafft sofort ein Ambiente, in dem man sich wohlfühlt. Alexander bezeichnet dieses Pattern als »a window place «:

»As a rule, a room that does not have a window place lacks quality; its windows are just holes in the wall. [...] Therefore: In every room where you spend any length of time during the day, make at least one window into a >window place $<\ll$ (Alexander 1977, 833ff.).

Ein Pattern trägt dazu bei, Spannungsfelder, die sich bei der Gestaltung ergeben, zu erkennen und zu lösen. Wenn ein Pattern die Auflösung von Spannungen ermöglicht, führt dies zu einer wahrnehmbaren $\gg$ subtle kind of freedom from inner contradictions (Alexander 1979). 


\subsection{Patterns of Integrated Organization als Entwicklungslandkarte}

Patterns sind eine Form der Analyse, mit welcher wir die Praxis durch Interpretation und Intervention wechselweise durchdringen können (Finidoori 2014). »Like diamonds, patterns are the result of many years of process. We don't make them - we find them, polish them, use them, and value them [...]. Once mined, observations can be captured as abstractions, visualizations « (West und Quillien 2015).

Die Patterns of Integrated Organization sind das Ergebnis jahrelanger Beobachtung und Reflexion im Umfeld (agiler) Organisationsentwicklung und sollen zum Explorieren einladen. Sie stellen eine Entscheidungshilfe und Landkarte zur Einführung neuer Arbeitsweisen dar. Sie schärfen die Gestaltung bewusster Beziehungsformen im Übergang von dominatorischen zu partnerschaftlichen Machtstrukturen (Eisler 1988) und helfen beim schrittweisen Aufbau von sicheren Räumen für eine gelingende Transformation.

\subsection{Das Core-Pattern Reflexion}

In den folgenden Abbildungen wird ein Ausschnitt aus der Pattern-Sammlung vorgestellt. Das Pattern //Reflexion// ist das Core-Pattern und stellt den Ausgangspunkt jeglicher Entwicklung dar. Die darin beschriebene Haltung ist Voraussetzung für die Arbeit mit der gesamten Patternsammlung.

Das Pattern enthält Fragen, welche die Bewusstwerdung über die eigene tatsächliche und gewünschte individuelle und kollektive Reflexionspraxis anregen und den Organisationen helfen, grundlegende Themen zu adressieren, die sich bei der Einführung einer bewussten Praxis eines strukturierten Reflexionsprozesses stellen.

Diese Reflexion ist eine rückwärts- oder nach innen gerichtete Aufmerksamkeit (vgl. Fraefel 2017, 59), die sich auf das Befremdende, Irritierende und Ungelöste in äußeren Handlungen und inneren Empfindungen richtet. Durch die Wahrnehmung des Störenden können die eigenen Konstrukte und Projektionen erkannt und potenziell bewusst gemacht werden. Die Wahrnehmung allein reicht jedoch nicht aus, um einen Reflexionsprozesses auszulösen. Es bedarf einer »Problemsensitivität « (Fraefel 2017, 60) und eine damit einhergehende Bereitschaft, das Ungelöste zu betrachten. Ist diese persönliche Verortung explizit gemacht, kann dies zu einer wirklichen Veränderung organisationaler Strukturen führen. Abbildung 1 enthält einen Ausschnitt aus dem Pattern //Reflexion//. 
Wie gelingt es Menschen, sich im Unternehmenskontext in Reflexion zu üben und mit sich und den anderen in eine radikal ehrliche Konversation zu treten?

Teams und soziale Gruppen in Unternehmen tendieren dazu Gruppenidentitäten zu schaffen, soziale Codes zu entwickeln und sich in in-groups und out-groups einzuteilen.

Wie gelingt es einer Gruppe, die Einzelnen in ihrer Einzigartigkeit wahrzunehmen, sich gegenseitig zu fördern und sich trotzdem als integrierte Gruppe zu sehen?

Wie kann eine Gruppe ein gegenseitiges Vertrauen und die Fähigkeit des Zuhörens entwickeln und sich als Resonanzkörper im Reflexionsprozess unterstützen?

DEMNACH braucht es eine Umgebung und einen Umgang, der auf gegenseitigem Vertrauen, Offenheit und Beziehungssicherheit aufbaut. Es müssen moderierte, zeitliche Räume zur Einübung von Reflexion zur Verfügung gestellt werden.

\section{Die Reflexion kann wie folgt strukturiert werden:}

1. Erstaunen, Verwirrung, Zweifel in einer ungelösten Situation

2. Wahrnehmen und Dokumentieren (Darstellen) mit allen Sinnen der inneren Empfindungen und äusseren Handlungen

3. Sorgfältige Analyse in Bezug des eigenen Selbstkonzeptes, der Motivation und Abwehrmechanismen

4. Imaginieren einer hypothetischen Umsetzung unter Einbezug der neuen Erkenntnisse aus der Analyse

5. Umsetzen der imaginierten Hypothese und Überprüfung der Richtigkeit in der Handlung

6. Reflexion bezüglich der neuen Empfindungen. Anpassung und Rückführung der neuen Erkenntnisse aus den Handlungen in das eigene Selbst-und Weltverständins

In vielen Situationen empfiehlt es sich das - Pattern FACILITATION einzusetzen. Der Aufbau von Beziehungssicherheit und Vertrauen ist besonders in der Zusamenrarbeit - Pattern MEETINGPROZESSE wichtig.

$>$ Weiterführende Informationen und Literaturliste

\section{Abb. 1: Ein Ausschnitt aus dem Pattern //Reflexion// (๑ Punkt.Null Organisationsent- wicklung)}




\subsection{Der Aufbau der Patternsammlung}

Die Patternsammlung legt den Fokus auf die Spannungsfelder, die in Unternehmen entstehen, welche neue, integrale, vermehrt selbstorganisierte Organisationsformen und Arbeitsweisen einführen wollen. Sie bieten jedoch auch Unternehmen, die sich nur informieren und unter Umständen mit einzelnen Patterns Erfahrungen sammeln möchten, eine relevante Orientierungshilfe.

Die Core-Patterns im innersten Kreis (vgl. Abb. 2) stellen das zentrale Instrument für eine Organisation dar, die über eine Neugestaltung nachdenkt. Mit der oben beschriebenen Reflexionspraxis können die weiteren Fragen, die sich bei der Gestaltung der Beziehungen ergeben, geklärt werden, zum Beispiel die Frage, wie Entscheide getroffen oder Spannungen und Konflikte geregelt werden. Diese sind in den Patterns of Interaction beschrieben. Die Patterns of Social Structure and Organization behandeln Fragen zum Aufbau, zur Struktur und Ausrichtung der Organisation, zum Beispiel Fragen zu Macht und Mitgliedschaft. Mit den Patterns of Organizational Processes werden Prozesse und Aktivitäten rund um die Kernaufgaben des Unternehmens geklärt, zum Beispiel nach welchem System die Entlöhnung erfolgt.

Die Abfolge, in der die Patterns zur Anwendung kommen, ist besonders wichtig. $\gg$ The key is getting the sequence of the decision right « (Quilien 2007). Alle Interventionen verändern das Feld der Kräfte und es besteht nicht immer die Möglichkeit, Interventionen rückgängig zu machen. Eine sinnvolle Abfolge der Patterns ist besonders relevant bei Transformationen, die einen Paradigmenwechsel anstreben. In Abbildung 3 sind zwei Beispiele einer solchen Sequenz von Entscheidungen dargestellt.

Beispiel 1 (roter Pfad): Wenn ein Unternehmen Hierarchien reduziert und Selbstorganisation einführt, stellt sich auch die Frage nach der Lohnstruktur. Wie wird geregelt, wer wieviel Lohn verdient?

$>$ Ebene Core-Patterns: Mit den Core-Patterns etabliert ein Unternehmen eine Reflexionspraxis, wie sie in den Patterns //Reflexion//, //Wahrnehmung// und //Imagination// beschrieben ist.

$>$ Ebene Patterns of Interaction: In einem ersten Schritt müssen sich die Mitglieder eines Unternehmens das Unternehmen bewusst machen, in welchen dominatorischen (Eisler 1988) oder kollektiv-partnerschaftlichen Macht- und Entscheidstrukturen sie zum aktuellen Zeitpunkt agieren. Danach gilt es gemeinsam zu imaginieren, wie kollektiv-partnerschaftlich oder dominatorisch sie sich zukünftig strukturieren wollen. Zur Unterstützung der Beantwortung dieser Frage dient das Pattern //Entscheidprozesse//. Wenn diese Bewusstwerdung erfolgt ist, kann die Organisation entsprechende Entscheidprozesse definieren, die es ermöglichen, kollektiv mit den gleichen Spielregeln für alle Organisationsmitglieder Entscheide zu treffen und individuelle Entscheide auf unterschiedliche Rollen zu verteilen. 


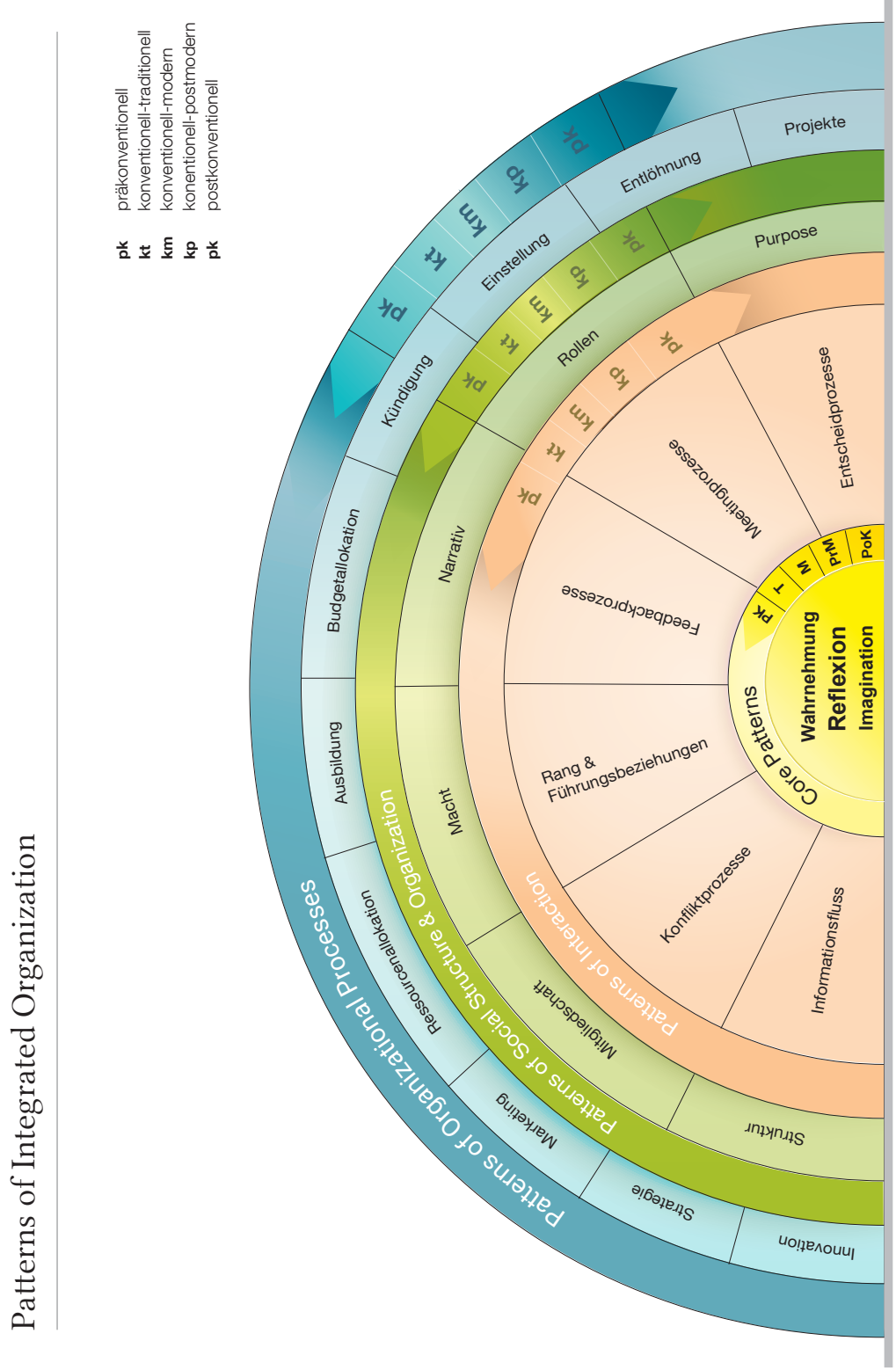

Abb 2: Das Modell der Patterns of Integrated Organization (๑ Punkt.Null Organisationsentwicklung) 


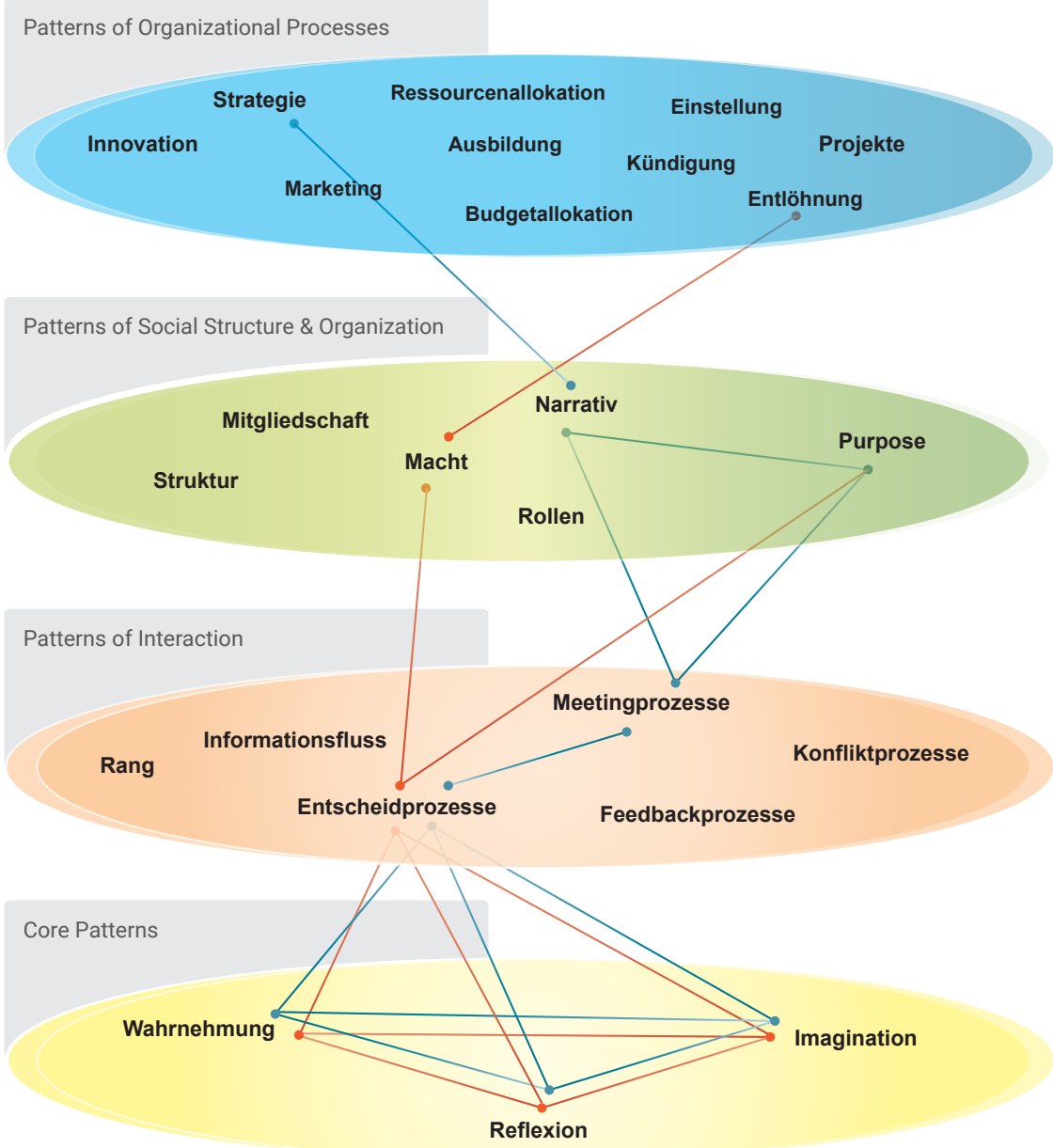

Abb. 3: Die vier Ebenen der Patterns of Integrated Organization (๑ Punkt.Null Organisationsentwicklung)

$>$ Ebene Patterns of Social Structure and Organization: Die definierten Entscheidprozesse können danach im Pattern //Macht// verbindlich geregelt und im Alltag anhand dieser Vereinbarung durchgesetzt werden. Im Falle eines Entscheides für eine selbstorganisierte Form steht die Macht nun nicht mehr nur Vorgesetzten als Vertreter_innen einer Hierarchie zu, welche diese nach Gutdünken einsetzen können, sondern sie wird an eine Verfassung abgegeben, in der geregelt ist, wie die Entscheidautorität verteilt ist. 
$>$ Ebene Patterns of Organizational Processes: Auf Basis dieser transparent festgehaltenen Entscheid- und Machstrukturen kann als Nächstes gemäß den Leitfragen und Praxisbeispielen im Pattern //Entlohnung// eine angemessene Lohnstruktur definiert werden. Die unterschiedlichen Bereiche von Verantwortung und die kollektivpartnerschaftliche Zusammenarbeit bilden dann die Grundlage für eine neu definierte Lohnstruktur.

Beispiel 2 (blauer Pfad): Wenn ein Unternehmen, das selbstorganisierte Strukturen einführt, eine Strategie festlegen will, muss es zuvor grundlegende Fragen klären.

$>$ Ebene Core-Patterns: Mit den Core-Patterns wird zuerst eine Reflexionspraxis etabliert, mit der sich die Organisation bewusst macht, worauf sie sich aktuell ausrichtet. Dabei reflektiert sie mithilfe des Patterns //Wahrnehmung// nicht das ideale Leitbild, sondern das, was zum aktuellen Zeitpunkt im Unternehmensalltag tatsächlich handlungsleitend ist. In dieser Reflexion wird transparent gemacht, wie der implizierte und der institutionalisierte Prozess stattfindet, mit dem diese Ausrichtung gefunden und überprüft wird. Mit der Unterstützung des Patterns //Imagination// wird danach gemeinsam reflektiert, wie dies in Zukunft geschehen soll.

$>$ Ebene Patterns of Interaction: Auf Basis dieser Erkenntnis kann eine Organisation mithilfe des Patterns //Entscheidprozesse// die Entscheidpraktiken festlegen. Dann gilt es mithilfe des Patterns //Meetingprozesse// zu entscheiden, in welchen Gefäßen der Austausch etabliert werden soll.

$>$ Ebene Patterns of Organizational Processes: In einem nächsten Schritt kann die Ausrichtung kollektiv expliziert und die emergente Entscheidung über die Ausrichtung gemeinsam angenommen werden. Auf diese Weise bildet sich ein evolutionärer //Purpose// als grundlegende Ausrichtung heraus und ein //Narrativ//, das sich regelmäßig weiterentwickelt.

$>$ Ebene Patterns of Organizational Processes: Geleitet von diesem kollektiv geteilten »Purpose « können mit der Unterstützung des Patterns //Strategie// konkrete Strategien abgeleitet werden, welche die Realität von unvereinbaren Spannungsfeldern auf dem Weg zum »Purpose« anerkennen und den Organisationsmitgliedern einen Wegweiser für den konkreten Umgang mit diesen Spannungsfeldern bieten.

In jedem Pattern der Patternsammlung findet sich ein Instrument, welches wir in Anlehnung an die Selbstentwicklungstheorie, einer »Psychologie der menschlichen Bedeutungsbildung «, von Cook-Greuter (2013) entwickelt haben. 

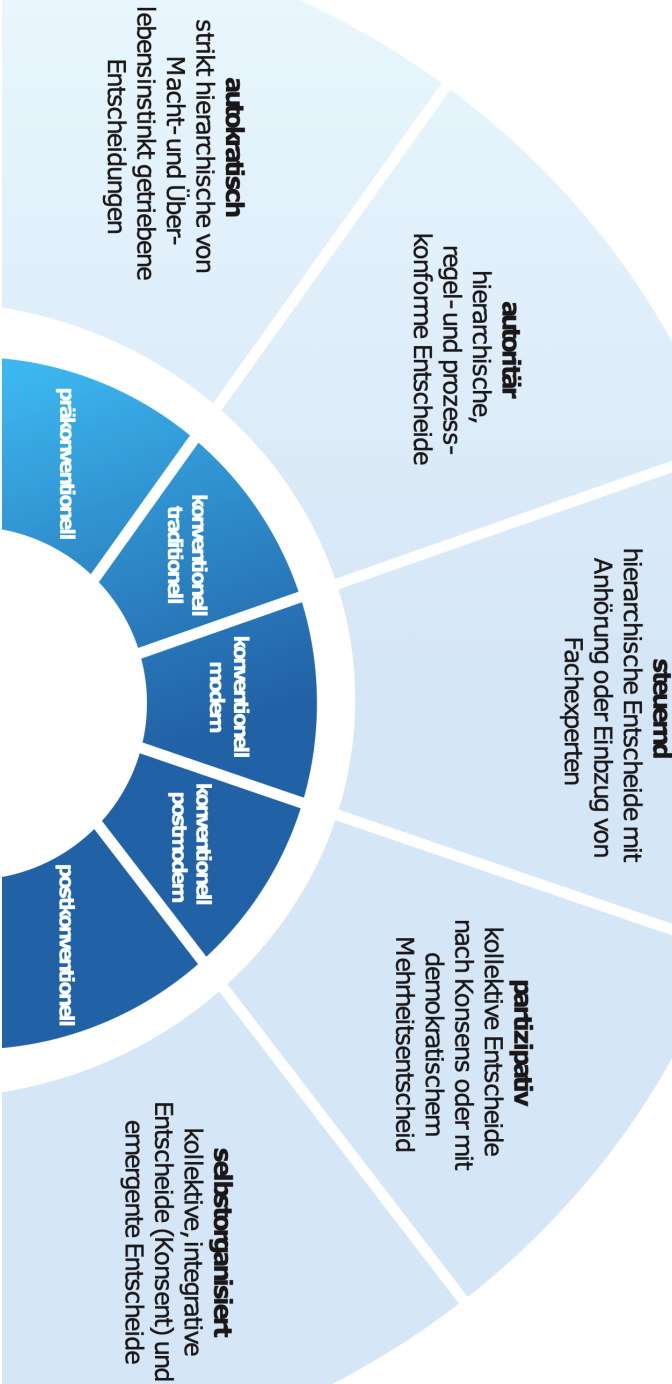

Abb. 4: Reflexionsinstrument am Beispiel des Patterns //Entscheidprozesse// (@ Punkt.Null Organisationsentwicklung) 
Dieses dient dazu, den gemeinsamen Ausgangspunkt wahrzunehmen, die erwünschte zukünftige Ausrichtung zu imaginieren und die gemeinsamen Praktiken zu entwickeln, welche diese zukünftige Ausrichtung ermöglichen. Damit haben die Unternehmen einen Kompass zur Hand, mit dessen Hilfe sie differenziert entscheiden können, wie sie die Qualität ihrer Zusammenarbeit und die Organisation der Zukunft gestalten können, in der sie sich frei fühlen $-\gg>$ at home $<$ in their world $\ll$.

\section{Resümee}

Seit mehr als drei Jahrzenten dient der Agilitätsbegriff als Projektionsfläche für einen bewegten Aufbruch und eine Neuordnung traditioneller Organisationen. In einer Welt und einer Zeit, die als äußerst bewegt erfahren wird, bedarf es dieser grundlegenden Fähigkeit zu Mitbewegung und Anpassung. Über die Bewegungsrichtung gibt die kognitiv-metaphorische Struktur des Begriffs keine Auskunft. Der Agilitätsbegriff lässt alle Projektionen zu und kann eine erfolgreiche, leistungsorientierte Organisation genauso versprechen wie kollaborative und vertrauensvolle Zusammenarbeitsformen von Peers oder eine sinnerfüllte Arbeitswelt. Doch um Raum für die Umsetzung der jeweils projizierten Sehnsucht zu schaffen, muss der eigene Agilitätsbegriff mit seinen Bedeutungsschreibungen und seiner Bedeutungsumgebung bewusst gemacht werden.

Dazu braucht es einen strukturierten Reflexionsprozess. In einer organisationalen Neugestaltung ist jeder Einzelne gefordert, ein Verständnis für sich selbst, für seinen Sprachgebrauch und seine Weltkonstruktion zu gewinnen. Diese Reflexion des eigenen Standpunktes findet immer auch im Zusammenspiel mit einem kollektiven Reflexionsprozess statt. Dabei gilt es nicht ein kollektives Einverständnis zu schaffen, sondern die eigene Ambiguitätstoleranz in Bezug zu den anderen und der gemeinsamen Transformation zu erhöhen.

An diesem Ausgangpunkt, in dem wir den Blick auf uns richten, ist Stillstand kein Stillstand der Nicht-Agilität, sondern ein lebendiger »Mittelzustand « (Noack 2020). Dieser wird mittels Reflexion zu unserem eigenen, individuellen Standpunkt, von dem aus wir mit der Gestaltung der Organisationen der Zukunft beginnen können.

\section{Anmerkungen}

1 Wahrig Herkunftswörterbuch A-Z. https://www.wissen.de/search?keyword=agil (Zugriff 30.12.2020).

2 DWDS. https://www.dwds.de/wb/agil (Zugriff 30.12.2020).

3 Macmillandictionary. https://www.macmillandictionary.com/dictionary/british/agile (Zugriff 30.12.2020).

4 Patternlanguage.com 2001-2020. https://www.patternlanguage.com (Zugriff 30.12.2020).

5 Patternlanguage.com 2001-2020. https://www.patternlanguage.com (Zugriff 30.12.2020). 


\section{Literatur}

14th Annual State of Agile Report. 2020. Zugriff 30.12.2020. https://stateofagile.com/\#ufh-i -615706098-14th-annual-state-of-agile-report/7027494.

Aeppli, Jürg und Hanni Lötscher. 2017. „Charakterisierung der Reflexionskategorien zum Rahmenmodell für Reflexion EDAMA«. In Reflexive Lehrerbildung revisited. Traditionen - Zugänge Perspektiven, hrsg. v. Constanze Berndt, Thomas Häcker und Tobias Leonhard, 159-175. Bad Heilbrunn: Julius Klinkhardt.

Alexander, Christopher. 1979. The Timeless Way of Building. New York: Oxford University Press.

Alexander, Christopher, Sara Ishikava, Murray Silverstein, Max Jacobson, Ingrid Fiksdahl-King und Angel Shlomo. 1977. A Pattern Language. Towns Buildings Construction. New York: Oxford University Press.

Baecker, Dirk. 2017. »Agilität, Hierarchie und Management: Eine Verallgemeinerung«. Universität Witten/Herdecke. Zugriff 30.12.2020. http://81.169.143.104/archiv/Empowerment\%20in\% 20Unternehmen/agilitaet_hierarchie_management1.pdf.

Beck, Kent, Mike Beedle, Arie van Bennekum, Alistair Cockburn, Ward Cuningham, Martin Fowler, James Grenning, Jim Highsmith, Andrew Hunt, Ron Jeffries, Jon Ker, Brian Marick, Robert C. Martin, Steve Mellor, Ken Schwaber, Jeff Sutherland und Dave Thomas. 2001. Manifesto for Agile Software Development. Zugriff 30.12.2020. https://agilemanifesto.org/.

Beedle, Mike. 2017. »Agile Uprising Podcast. Manifesto Author Review«. Interview von Ryan Lockard und James Gifford. 10. März 2017. Audio, 00:06:56-00:07:28 und 00:19:51-00:22:03. Zugriff 30.12.2020. http://podcast.agileuprising.com/manifesto-author-review/.

Bröckling, Ulrich. 2014. »Wettkampf und Wettbewerb. Semantiken des Erfolgs zwischen Sport und Ökonomie«. Leviathan 42 (Sonderband 29/2014): 71-81.

Brückner, Fabian und Falko von Ameln. 2016. »Agilität«. Wiesbaden: Springer Fachmedien. Online publiziert: 9. September 2016. https://doi.org/10.1007/s11612-016-0334-6.

Cockburn, Alistair. 2002.»Agile Software Development«. The AgileSoftware Development Series. Cockburn*Highsmith Series Editors. Draft Version: 3b. Zugriff 30.12.2020. https://www.academia. edu/5767518/Agile_Software_Development_The_Agile_Software_Development_Series_ Cockburn_Highsmith_Series_Editors.

Combe, Arne und Ulrich Gebhard. 2012. Verstehen im Unterricht. Die Rolle von Fantasie und Erfahrung. Wiesbaden: Springer Verlag.

Cook-Greuter, Susanne R. 2010. Postautonomous Ego Development: A Study of Its Nature and Measurement. Diss., Harvard University Graduate School of Education.

Cook-Greuter, Susanne R. 2013. Nine Levels Of Increasing Embrace In Ego Development: A Full-Spectrum Theory Of Vertical Growth And Meaning Making. Zugriff 30.12.2020. https://www. semanticscholar.org/paper/Nine-Levels-Of-Increasing-Embrace-In-Ego-\%3A-A-Theory -Cook-Greuter/cc0e81e8aaf82e6ec4faccbc3ed9889fe0cd2bb7.

Cunningham, Ward und Michael W. Mehaffy. 2013. Wiki as Pattern Language. Sustasis Foundation. Zugriff 30.12.2020. https://dl.acm.org/doi/pdf/10.5555/2725669.2725707.

De Meyer, Arnoud und Kasra Ferdows. 1991. »Removing the barriers in manufacturing: The 1990 European manufacturing futures survey«. European Management Journal 9 (1): 22-29.

Denning, Steve. 2019. »Understanding Fake Agile«. Forbes, 23. Mai. Zugriff 30.12.2020. https://www. forbes.com/sites/stevedenning/2019/05/23/understanding-fake-agile/?sh=3c975c424bbe.

Deutscher, Guy. 2011. Du Jane, ich Goethe. Eine Geschichte der Sprache. München: DTV.

Dove, Rick. 1992. The 21st Century Manufacturing Enterprise Strategy or What Is All This Talk About Agility? lacocca Institute, 1991. Zugriff 30.12.2020. http://www.parshift.com/Files/PsiDocs/ Rkd92Art6.pdf. 
Dove, Rick. 1994. The Meaning of Life \& The Meaning of Agile. Zugriff 30.12.2020. https://www. researchgate.net/publication/284663148_The_meaning_of_life_and_the_meaning_of_agilen.

Eisler, Rianne. 1988. The Chalice and the Blade. New York: Harper Collins.

Finidori, Hélène. 2014. A Pattern Language for Systemic Transformation (PLAST) (re)Generative of Commons. Konferenz »Purplsoc Workshop - The Pursuit of Pattern Languages for Societal Change«.

Förster, Kerstin und Roy Wendler. 2012. Theorien und Konzepte zu Agilität in Organisationen. Dresdner Beiträge zur Wirtschaftsinformatik Nr. 63/12. Dresden: Technische Universität Dresden.

Fowler, Martin. 2005. Semantic Diffusion. 14.12.2005. Zugriff 30.12.2020. https://martinfowler.com/ bliki/SemanticDiffusion.html.

Fowler, Martin. 2018. The State of Agile Software in 2018. Transcription of my keynote at Agile Australia. 25.08.2018. Zugriff 30.12.2020. https://martinfowler.com/articles/agile-aus-2018.html.

Fraefel, Urban. 2017. „Wo ist das Problem? Kernideen des angloamerikanischen Reflexionsdiskurses bei Dewey und Schön«. In Reflexive Lehrerbildung revisited. Traditionen - Zugänge Perspektiven, hrsg. v. Constanze Berndt, Thomas Häcker und Tobias Leonhard, 56-73. Bad Heilbrunn: Julius Klinkhardt.

Goldmann, Steven L. 1994. Agile Competitors and Virtual Organizations: Strategie for Enriching the Customer. New York: John Willy and Sons.

Grenning, James. 2017. Agile Uprising Podcast. Manifesto Author Review. Interview vom 10. März 2017. Audio. Zugriff 30.12.2020. http://podcast.agileuprising.com/manifesto-author-review/.

Hacking, lan. 2001. „Vom Gedächtnis der Begriffe«. In Was ist ein philosophisches Problem?, hrsg. v. Joachim Schulte und Uwe Justus Wenzel, 72-86. Frankfurt a. M.: Fischer TB Verlag.

Hamacher, Katharina. 2018. „Wer überleben will, sollte auf Veränderung setzen«. Handelsblatt, 19. September. Zugriff 30.12.2020. https://www.handelsblatt.com/autoren/hamacher -katharina/22838022.html?ticket=ST-3187081-BuYSGZ76YYvIABgakUfS-ap5.

Highsmith, Jim. 2001. Agile Alliance. Zugriff 30.12.2020. https://agilemanifesto.org/history.html.

Hilzensauer, Wolf. 2017. Wie kommt die Reflexion in den Lehrerberuf? Ein Lernangebot zur Förderung der Reflexionskompetenz bei Lehramststudierenden. Münster: Waxmann.

Johansen, Françoise und Suzanne Van den Bosch. 2017. »The scaling up of Neighbourhood care: From experiment towards a transformative movement in healthcare«. Futures 89: 60-73. https://doi.org/10.1016/j.futures.2017.04.004.

Joiner, Bill und Stephen Josephs. 2007. Leadership Agility. Five Levels of Mastery for Anticipating and Initiating Change. San Francisco: Jossey-Bass/Wiley. Kindle.

Karlsson, Christer. 1989. »High rates of innovation: The Japanese culture shock to Europe«. European Management Journal 7 (1): 31-39.

Kegan, Robert. 1994. In Over Our Heads. The Mental Demands of Modern Life. Cambridge, MA: Harvard University Press.

Komus, Ayelt. 2020. »Status Quo Agile 2019/2020. Ergebnisbericht. 4. Internationale Studie zu Nutzen und Erfolgsfaktoren (skalierter) agiler Ansätze«. Zugriff 30.12.2020. www.status-quo-agile.de.

Koplowitz, Herb. 1984. »A projection beyond Piaget's formal operations stage: a general system stage and a unitary stage «. In Beyond formal operations, hrsg. v. Michael L. Commons, Francis A. Richards und Cheryl Armon, 272-296. New York: Praeger.

Korthagen, Fred A.J. 2014. »Promoting core reflection in teacher education: Deepening professional growth«. In International Teacher Education: Promising pedagogies (Part A), hrsg. v. Lilly Orland-Barak und Cheryl J. Craig, 73-89. Bingley, UK: Emerald.

Kühl, Stefan. 2017. ")Die agile Organisation ist kalter Kaffees. Interview von Jan C. Weilbacher«. Human Resources, 16. Januar. Zugriff 30.12.2020. https://www.humanresourcesmanager.de/ news/die-agile-organisation-ist-kalter-kaffee.html. 
Kühl, Stefan. 2019. Die überraschende Renaissance eines verstaubten soziologischen Konzeptes. Wie Praktiker das Wort »agil« missverstehen. Working Paper 2/2019. Zugriff 30.12.2020. https:/ /www.uni-bielefeld.de/soz/personen/kuehl/pdf/Working-Paper-2-2019-Die-uberraschende -Renaissance-eines-verstaubten-soziologischen-Konzeptes.pdf.

Lakoff, George und Mark Johnson. 1980. Metaphors we live by. Chicago: University of Chicago Press. Kindle.

Lakoff, George und Mark Johnson. 1999. Philosophy in the flesh: The embodied mind and its challenge to western thought. New York: Basic Books. Kindle.

Lakoff, George und Mark Johnson. 2003. »Afterword«. In Metaphors we live by, hrsg. v. George Lakoff und Mark Johnson, 243-76. Chicago: University of Chicago Press. Kindle.

Laloux, Frederic. 2014. Reinventing Organizations. A Guide to Creating Organizations Inspired by the Next Stage of Human Consciousness. Brussels: Nelson Parker. Kindle.

Laloux, Frederic. 2015. Reinventing Organisations. Ein Leitfaden zur Gestaltung sinnstiftender Formen der Zusammenarbeit. Übersetzt von Mike Kauschke. München: Verlag Franz Vahlen.

Lutter, Andreas. 2016. »Metaphern und Analogien der Wirtschaft. Konzeptuelle Wirtschaftsmetaphorik und ihre Bedeutung für ökonomisches Lernen«. Zeitschrift für ökonomische Bildung 16 (5): 150-68. Zugriff 30.12.2020. https://www.zfoeb.de/2016_5/2016_5_lutter_metaphern_ und_analogien_der_wirtschaft.pdf.

Majkovic, Anna-Lena, Ellen Gundrum, Sabine Maria Benz, Nora Dzsula und Rafael Huber. 2019. IAP Studie 2019: Agile Arbeits- und Organisationsformen in der Schweiz. Ergebnisse der qualitativen Interviews. Zugriff 30.12.2020. https://www.zhaw.ch/storage/psychologie/upload/iap/ studie/IAP-Studie_Agilitaet_2019.pdf.

Marick, Brian und informIT Editorial Team. 2011. Ten Years of Agile: An Interview with Brian Marick. Zugriff 30.12.2020. https://www.informit.com/articles/article.aspx?p=1739477.

Maurer, Peter und Kerstin Treutner. 2020. »Was macht einen Prozess agil - und was bringt das? «. In Agilität? Herausforderungen neuer Konzepte der Selbstorganisation, hrsg. v. Stefanie PorschenHueck, Marc Jungtäubl und Margit Weihrich, 49-60. Augsburg: Rainer Hamp Verlag.

Morgan, Gareth. 1997. Bilder der Organisation. Stuttgart: Schäffer-Poeschel Verlag.

Moser, Karin S. 2000. Metaphern des Selbst. Wie Sprache, Umwelt und Selbstkognition zusammenhängen. Lengerich: Pabst Science Publishers. Kindle (Psychologia universalis, 23).

Nagel, Roger N. 1991.21st Century Manufacturing Enterprise Strategy Report. An Industry-Led View. lacocca Institute, Januar 1991. Zugriff 30.12.2020. https://www.researchgate.net/publication/ 239666989_21st_Century_Manufacturing_Enterprise_Strategy-an_Industry_led_View.

Newton, John. (2017). Wer agil ist, überlebt! Zugriff 30.12.2020. https://www.silicon.de/blog/wer -agil-ist-ueberlebt.

Noack Napoles, Juliane. 2020. »Identität als Stillstand. Ein metaphernanalytischer Blick auf eine Nicht-Bewegung«. In Bewegungen. Beiträge zum 26. Kongress der Deutschen Gesellschaft für Erziehungswissenschaft, hrsg. v. Isabell van Ackeren, Helmut Bremer, Fabian Kessl, Hans Christoph Koller, Nicolle Pfaff, Caroline Rotter, Dominique Klein und Ulrich Salaschek, 505-13. Opladen, Berlin \& Toronto: Verlag Barbara Budrich.

Piaget, Jean. 1983. Meine Theorie der geistigen Entwicklung. Frankfurt a. M.: Fischer Taschenbuch Verlag. Pielenz, Michael. 1993. Argumentation und Metapher. Tübingen: Gunter Narr Verlag.

Porschen-Hueck, Stefanie, Marc Jungtäubl und Margit Weihrich, Hrsg. 2020. Agilität? Herausforderungen neuer Konzepte der Selbstorganisation. Augsburg: Rainer Hamp Verlag.

Quartel, Ron. 2019. Teal is the New Agile. Zugriff 30.12.2020. https://ronquartel.medium.com/teal -is-the-new-agile-3d0f56d04d54.

Quillien, Jenny. 2007. „Grasping the Ineffable: From Patterns to Sequences«. Environmental \& Architectural Phenomenology 18: 4-10. 
Rigotti, Francesca. 1994. Die Macht und ihre Metaphern: über die sprachlichen Bilder der Politik. Frankfurt a. M.: Campus Verlag.

Robertson, Brian J. 2015. Holacracy. the new management system for a rapidly changing world. New York: Henry Holt.

Rosa, Hartmut. 2013. Beschleunigung und Entfremdung. Berlin: Suhrkamp Verlag.

Schieder, Siegfried. 2006. Die gestaltende Kraft von Sprachbildern und Metaphern. Deutungen und Konstruktionen von Staatlichkeit in der deutschen Debatte über den europäischen Verfassungsvertrag. Diss., Universität Trier.

Schmitt, Rudolf. 2017. Systematische Metaphernanalyse als Methode der qualitativen Sozialforschung. Wiesbaden: Springer Fachmedien.

Schmitz-Ohrndorf, Martin. 2016. »Stillstand ist der Tod«. EntwicklerMagazin 4.Zugriff30.12.2020.https ://kiosk.entwickler.de/entwickler-magazin/entwickler-magazin-4-2016/stillstand-ist-der-tod/.

Sherehiy, Bohdana und Waldemar Karwowski. 2007. »A Review of Enterprise Agility: Concepts, Frameworks, and Attributes«. International Journal of Industrial Ergonomics 37 (5): 445-60.

Sutherland, Jeff. 2011. Takeuchi and Nonaka: The Roots of Scrum. Blog vom 22.11.2011. Zugriff 30.12.2020. https://www.scruminc.com/takeuchi-and-nonaka-roots-of-scrum/.

Sutherland, Jeff. 2014. The Key Agile Principles Behind Holacracy. Blog vom 13.02.2014. Zugriff 30.12.2020. https://openviewpartners.com/blog/agile-principles-behind-holacracy/\#. YBecNuhKg2x.

Takeuchi, Hirotaka und Ikujiro Nonaka. 1986. »The New New Product Development Game«. Harvard Business Review (January 1986). Zugriff 30.12.2020. https://hbr.org/1986/01/the-new -new-product-development-game.

Tisdale, Tim. 1998. Selbstreflexion, Bewusstsein und Handlungsregulation. Weinheim: Psychologie Verlags Union.

Truscheit, Karin. 2018. „Unsere Unworte des Jahres«. Frankfurter Allgemeine Zeitung, faz.net, 31. Dezember. Zugriff 30.12.2020. https://www.faz.net/aktuell/karriere-hochschule/buero -co/sprache-im-buero-unsere-unworte-des-jahres-15953965-p3.html.

Torbert, Bill. 2004. Action Inquiry. The Secret of Timely and Transforming Leadership. San Francisco: Berret-Kohler Publishers. Kindle.

Vlissides, John M., James O. Coplien und Norman L. Kerth, Hrsg. 1996. Pattern Languages of Program Design. Reading, Massachusetts: Addison Wesley Longman, Inc.

West, David und Jane Quillien. 2015. The mystery Case of Undiscovered Patterns. Konferenzpapier zur »Purplsoc Conference 2015«. Zugriff 30.12.2020. https://hillside.net/plop/2015/papers/ steelers/20.pdf.

Westphal, Kristin. 2014. »Bewegung«. In Handbuch Pädagogische Anthropologie, hrsg. v. Christoph Wulff und Jörg Zirfas, 146-53. Wiesbaden: Springer VS.

Wendler, Roy. 2013. »The Structure of Agility from Different Perspectives«. Federated Conference on Computer Science and Information Systems, 1177-84, September, 8-11. Zugriff 30.12.2020. https://ieeexplore.ieee.org/document/6644163.

\section{Die Autorinnen}

Franziska Espinoza begleitet seit mehr als zwei Jahrzehnten Teams und Organisationen in komplexen Veränderungs- und Konfliktsituationen, bei der Einführung selbstorganisierter Arbeitsformen und der Entwicklung integraler Führungskompetenz. Sie ist Mitgründerin von Punkt.Null GmbH.

Kontakt:www.punktnull.be, Franziska.Espinoza@punktnull.be 
Sabine Mommartz arbeitet als Dozentin für Fachdidaktik Musik an der PHBern und promoviert zurzeit in Frankfurt bei Prof. Dr. Maria Spychiger. Sie leitet Chöre und arbeitet freiberuflich als Coach bei der Firma Punkt Null GmbH. In ihrer Forschung beschäftigt sie sich hauptsächlich mit der pädagogischen Umsetzung der Selbstkonzeptgenese und dem Umgang mit Kontingenz.

Kontakt:Sabine.Mommartz@phbern.ch 